\title{
Canada Communicable Disease Report 2016 readership survey
}

\author{
P Huston ${ }^{1 *}$, NM Farrell², L Townley²
}

\begin{abstract}
Background: The Canada Communicable Disease Report (CCDR) is a peer reviewed scientific journal published since 1975. In 2011, a readership survey was conducted to inform a revitalization process. In late 2016 , this survey was repeated to assess progress.

Objective: To provide information about the results of the CCDR 2016 readership survey, which identified CCDR's readership and their needs, obtained feedback on the journal's revitalization and sought suggestions for further improvement.
\end{abstract}

Methods: An online readership survey was conducted from September 7 to 28, 2016. Invitations were sent via email to CCDR subscribers. The survey was based on the 2011 version and checked for face-validity. Analysis included descriptive statistics and a qualitative assessment of comments for themes.

Results: A total of 549 people responded to the survey (12\% participation rate). The majority of respondents worked in public health (61\%), clinical care (23\%), academia (16\%) and laboratory medicine (9\%). Approximately $45 \%$ of respondents had received CCDR for less than four years, which is consistent with the fact that the number of subscribers more than doubled over this time. Over $90 \%$ of respondents reported they read the articles in CCDR (always 15\%, often $43 \%$, sometimes $35 \%$ ). When asked about their primary source of infectious disease information in Canada, CCDR was the number one response, identified by $72 \%$ of respondents. When asked "What do you like best about CCDR?" typical comments were that it provided Canadian content, was well written, evidence-based, interesting and relevant. The number one suggestion for improvement was that CCDR should be listed with PubMed.

Conclusion: The survey results suggest that CCDR has been successfully revitalized and is meeting its readership's needs for a scientific journal on infectious disease with Canadian content, high quality and relevance. Consistent with suggestions for improvement, CCDR will be joining the PubMed database over the next year.

Suggested citation: Huston P, Farrell NM, Townley L. Canada Communicable Disease Report 2016 readership survey. Can Commun Dis Rep. 2017;43(2):56-8. https://doi.org/10.14745/ccdr.v43i02a04
Affiliations

${ }^{1}$ CCDR Editorial Office, Infectious Disease Prevention and Control Branch, Public Health Agency of Canada, Ottawa, ON

${ }^{2}$ Communications and Public Affairs Branch, Health Canada, Ottawa, ON *Correspondence: patricia.
huston@phac-aspc.gc.ca

\section{Introduction}

The Canada Communicable Disease Report (CCDR) is a scientific, peer reviewed, online, bilingual journal that has been in continuous publication since 1975. It was originally published by Health Canada and is now published by the Public Health Agency of Canada (PHAC). In 2011, following a decrease in the publication schedule, a CCDR readership survey was conducted to inform a possible renewal of the journal. At that time CCDR had approximately 2,000 subscribers. Readers noted that CCDR needed to reinvent itself and "bring back timely Canadian communicable disease-relevant articles". The 2011 survey respondents noted they wanted more early reports of outbreaks with potential to spread (95\%), national surveillance reports on notifiable diseases $(91 \%)$ and research articles on infectious disease topics of relevance to Canada (91\%) [unpublished data].

Following the 2011 survey, the editorial office was re-established and, based on the survey results, CCDR was revitalized and began publishing regular issues with peer reviewed articles in 2013. Its present mandate is to publish timely information on current and emerging infectious diseases of relevance to Canada to inform policy, program development and practice. The number of subscribers more than doubled between the
2011 and 2016 surveys. We have been doing monthly tracking of subscriptions since 2014. (Figure 1)

\section{Figure 1: Subscriptions to Canada Communicable Disease Report: January 2014-August 2016}

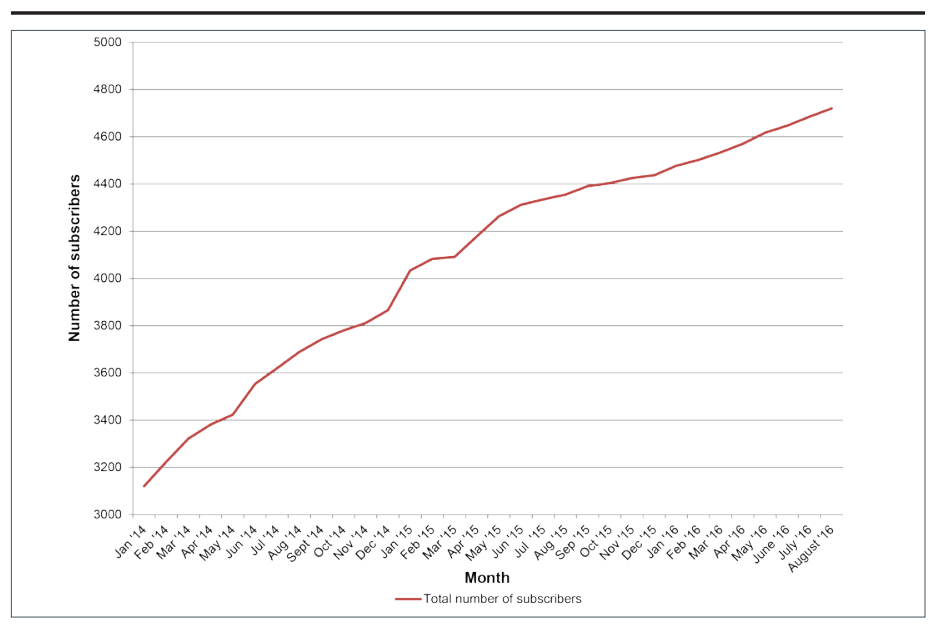


In the summer of 2016, the CCDR editorial office worked with Health Canada's Communication and Public Affairs Branch to refine and then repeat the readership survey. The objective of the 2016 survey was to identify CCDR's readership, obtain feedback on the journal post-revitalization and see how it can be further improved. This is a summary of the full Readership Survey Report (unpublished document, Communications and Public Affairs Branch, Health Canada).

\section{Methods}

Survey methods were based on the federal government online survey standards (1). The 2016 online survey was based on the 2011 survey and checked for face-validity. It included questions about the readership, how they liked the journal, how they received information on infectious diseases in general and how CCDR might be improved. Most of the survey questions were multiple choice format followed by a comments section which included a question about how to improve CCDR. Following pilot testing in both official languages, an invitation to complete the online survey was sent by email to CCDR's 4716 subscribers.

The survey was available online from September 7 to 28, 2016. Two reminders were sent to non-responders to optimize the participation rate. The participation rate was calculated by dividing the number of responders by the sum of responders, non-responders and the number of bounce-backs minus any email addresses found to be invalid. Analysis of the responses included descriptive statistics and a qualitative assessment of comments for themes.

\section{Results}

\section{Canada Communicable Disease Report's readership and information sources}

Of the 4716 subscribers, 17 emails were found to be invalid for a total of 4699 participants. Of those 549 surveys were completed for a participation rate of $12 \%$. The majority of respondents indicated they worked in public health (61\%), followed by clinical care $(23 \%)$, academia (16\%) and laboratory medicine $(9 \%)$. This total exceeds $100 \%$ because it was possible to select more than one response option (for example, clinical care AND academia). Slightly less than $20 \%$ of respondents were 35 years of age or younger, about $40 \%$ were between $36-50$ years old and $40 \%$ were over 50 years old. Almost $50 \%$ of respondents had been receiving CCDR for less than four years, which is consistent with the fact that the number of CCDR subscribers doubled since the 2011 survey.

Most readers were from Ontario and Quebec, although there were readers from almost every province and all of the territories, as well as from the United States, the European Union and elsewhere (Figure 2). Nearly half of all respondents noted they first heard about CCDR either at a conference or through a colleague or teacher.

The most common resources respondents used to obtain information about infectious diseases were websites (75\%), colleagues (72\%) and online literature searches (69\%). Other sources were noted in the comments such as ProMED. When
Figure 2: Canada Communicable Disease Report: Readership by location

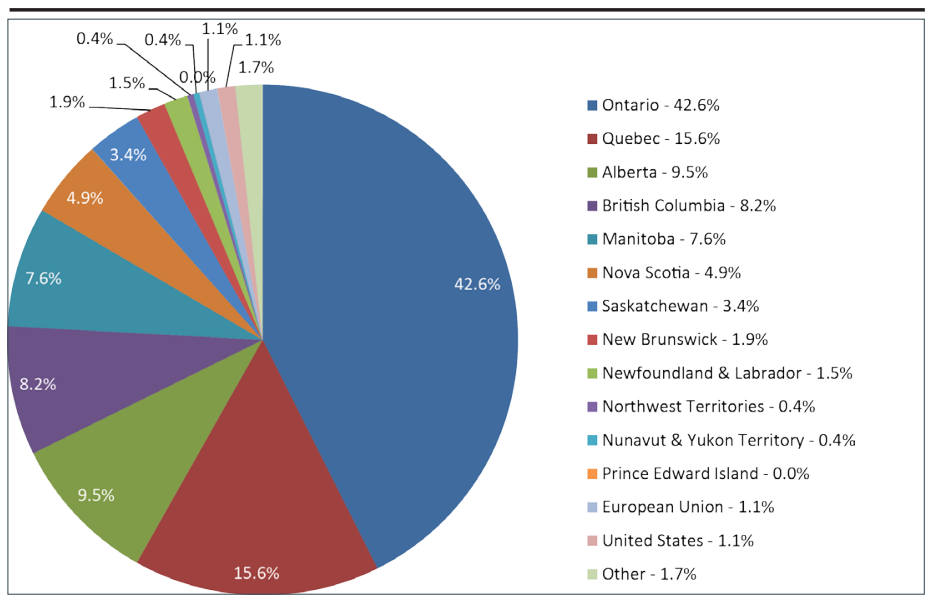

asked how they were most likely to obtain Canadian-specific information on infectious diseases and immunization, the top source was CCDR (72\%), followed by local public health (52\%), updates from province/territory (almost $50 \%$ ) and the National Advisory Committee on Immunization (NACl) (50\%) (Figure 3).

Figure 3: Response to 2016 Canada Communicable Disease Report survey question: "How are you most likely to get Canadian-specific information on infectious diseases and immunization (e.g. local outbreak, new vaccine indications)?" $(n=549)$

\begin{tabular}{|l|c|}
\hline CCDR & $72.3 \%$ \\
Local Public Health & $52.1 \%$ \\
National Advisory Committee on Immunization (NACI) & $49.5 \%$ \\
Update from my province or territory & $49.4 \%$ \\
Canadian Immunization Guide & $45.0 \%$ \\
Canada.ca/health & $31.1 \%$ \\
The media & $27.1 \%$ \\
Local expert & $20.0 \%$ \\
Other & $18.8 \%$ \\
No response & $2.0 \%$ \\
\hline
\end{tabular}

\section{Feedback on the revitalized Canada Communicable Disease Report}

Over $90 \%$ of respondents reported reading the articles in CCDR (always $15 \%$, often $43 \%$, sometimes $35 \%$ ) and found the following types of articles to be useful or very useful:

- Rapid communication on outbreaks with potential to spread (90\%)

- $\quad$ PHAC or advisory committee guidelines for communicable diseases (87\%)

- Research articles on infectious disease topics of relevance to Canada (86\%)

- National surveillance reports on notifiable diseases (84\%)

Comments were provided by $62 \%$ of respondents $(n=340)$ in the feedback section.

The most common strengths identified were CCDR's Canadian content, high quality and relevance. When asked "What do you like best about CCDR?" typical comments were:

"Canadian, well written, evidence based", "interesting articles", "topical", "relevant to my work", and "concise and reliable." 


\section{Suggestions for improvement and next steps}

When asked "What do you think is the most important way we can improve CCDR?" almost $50 \%$ of respondents commented $(n=266)$. The most frequent comments were: "keep up the good work" and "get indexed on PubMed/Medline". Other suggestions were:

- Reach out to students in universities and give presentations

- Include more foodborne outbreak reports

- Have more articles from authors across Canada

- Be as timely as possible especially with surveillance data and outbreak reports

When asked, $40 \%$ of respondents stated they would like to access CCDR using a tablet or smartphone app. However, if an app included CCDR and other resources, such as the Canadian Immunization Guide, the Sexually Transmitted Infection Guide and the Tuberculosis Standards, $46 \%$ of respondents stated they would download it. A typical comment was "I would like to be able to access CCDR from my iPhone."

\section{Discussion}

CCDR readers come from across Canada and around the world. Most respondents work in public health and clinical care. Readers like the CCDR's revitalized content, high quality and relevance and identify the journal as their top source for Canadian-specific infectious disease information. In 2011, CCDR readers asked for more rapid communications, summaries of advisory committee recommendations and research articles on infectious diseases. These types of articles have been published in CCDR over the last three years and $85-90 \%$ of readers have found them useful.

Since CCDR was revitalized, its subscription rate has more than doubled. Survey responders suggested more outreach, Canadian authors, increased timeliness and a mobile application. However, the most common suggestion for improvement was to list the journal with PubMed.

The key limitation of this survey is the response rate of $12 \%$. Although this is not unusual for an online survey, there is no assurance that the respondents are representative of the entire CCDR readership.

The readership survey confirms that CCDR has come a long way in the past five years and its readers find it to be their go-to source for practical, authoritative information on infectious diseases in Canada. We are pleased to announce that CCDR has passed the scientific review for PubMed and full text articles will be available through PubMed Central in 2017.

\section{Contributors}

Many thanks to the following people who assisted in the development of the survey and this article:

- $\quad$ Lidiya Tsegaye assisted with drafts of survey instrument

- Kyla Tyson provided the subscription data, conducted the analysis of survey comments and developed the initial draft of manuscript

- Mylène Poulin provided input on initial drafts of the survey instrument and drafts of the manuscript

\section{Acknowledgements}

Many thanks to all CCDR's contributors and to all the respondents of the CCDR survey.

\section{Conflict of interest}

Dr. Patricia Huston is the Editor-in-Chief of CCDR and recused herself from editorial decisions regarding this manuscript. The Associate Editor, Dr. Hilary Robinson, made the editorial decisions on this manuscript.

\section{Funding}

The CCDR is supported financially by PHAC.

\section{Reference}

1. Public Services and Procurement Canada. Standards for the conduct of Government of Canada public opinion research - online surveys [Internet]. Ottawa (ON): Government of Canada; [updated 2014 Nov 19; cited 2017 Jan 10]. Available from: https://www.tpsgc-pwgsc.gc.ca/rop-por/ enligne-online-eng.html\#s8. 